\title{
New SPR-based methods for analysis of allergenic agents used in wine treatment
}

\author{
Hauke Wessels and Angelika Paschke-Kratzin \\ Institute of Food Chemistry, University Hamburg, Grindelallee 117, 20146 Hamburg, Germany
}

\begin{abstract}
The use of allergenic agents in wines needs to be monitored by both producers and supervisory authorities for the protection of sensitive individuals. Currently established as the gold standard is the ELISAtechnique which relies on the affinity and specificity of antibodies. Since antibodies are produced in animals and current legislative developments demand the reduction of the use of animals for scientific purposes, the biosensor technology could be interesting for the quantification of allergens. By the combination of a technical device, the surface plasmon resonance, and a specific antibody it was possible to develop an antibody-based analytical method which is capable to detect lysozyme in quantities below $0.25 \mathrm{ppm}$. Since the biosensor is reusable multiple times, this approach can contribute to reduce the antibody consumption and therefore the use of animals for analysis. Furthermore, first insights in the use of new molecular receptors, aptamers were gained.
\end{abstract}

\section{Introduction}

An allergy is a reaction of the immune system to normally harmless substances, e.g. food components. Mostly proteins and glycoproteins are responsible for the adverse reactions like nausea, exanthema or in severe cases anaphylactic shocks [1]. After an initial contact, specific antibodies are produced by the immune system so that every following contact triggers the adverse reactions. Since a therapy is currently not available, the avoidance of the allergen is the only option for sensitive consumers [2]. So, to protect sensitive individuals, the EU legislation demands the labelling of food (including wine) which contains allergens listed in EU regulation 1169/2011 annex II, both as a trace or as an ingredient [3]. A labelling (use of terms or pictograms according to commission implementing regulation 579/2012) of ovalbumin and lysozyme in wine is therefore necessary since exceptions are no longer applicable [4]. According to EU regulation 1308/2013 in combination with OIV/OENO 427/2010 and OIV-COMEX 502-2012, an amount of allergens above $0.25 \mathrm{ppm}$ requires a labelling [5-7].

The ELISA-method (enzyme-linked immunosorbent assay) is commonly used for analysis of allergens; especially the sandwich-technique is capable of low limits of detection and quantification (LOD and LOQ) [8, 9]. Neither PCR- (polymerase chain reaction) nor MSmethods (mass spectrometry) are capable to detect the immunogenicity of allergenic residues, which is only possible by the use of antibody-based methods [10]. However, by the EU directive 2010/63 the use of animals for research should be reduced as much as possible. As the production of antibodies is done in animals (polyclonal antibodies) or animal-dependent cell culture (monoclonal antibodies) a reduction or elimination of antibodies in analytics should be aimed for. An option for this is either the development of new analytical methods with a reduced consumption of antibodies or a complete substitution by equivalent molecular receptors.

The development of an antibody-based biosensor could be an opportunity to reduce the necessary amount of antibodies [11]. Biosensors are devices which combine a molecular receptor with an electrical device so that a biological interaction can be translated into an electrical signal [12]. In contrast to ELISA-methods, where the used antibody is only taken for a single run, biosensors can be used multiple times so that the amount of the molecular receptor can be reduced significantly. In the presented work a SPR-spectroscope (surface plasmon resonance) was used as device to quantify the molecular interaction between a receptor and lysozyme.

The SPR-spectroscopy is a method which is capable to visualize and quantify interactions between one immobilized partner and one partner which is injected into a flow system [13]. The immobilization takes place on a thin gold film, which is located on a glass prism. Linear polarized light enters the prism and at a certain angle (SPR-angle) total reflection at the interface of prism and gold film occurs (see Fig. 1). Part of the energy of the light stimulates an evanescent field above the gold film and protrudes into the flow system, which leads to a decrease in the reflected light intensity. An interaction between the immobilized and the injected binding partners takes place in the area of the evanescent field, which is therefore influenced by them. This leads to a shift of the angle of total reflection which can therefore be used for analysis.

The use of artificial receptors which can be generated without the use of animals or animal-dependent technologies could be an option to replace antibodies overall. A possibility for this could be the so-called aptamers, single-stranded nucleic acids $[14,15]$. Due to the missing complementary strand, the DNA- or RNAmolecules form defined secondary structures which are 


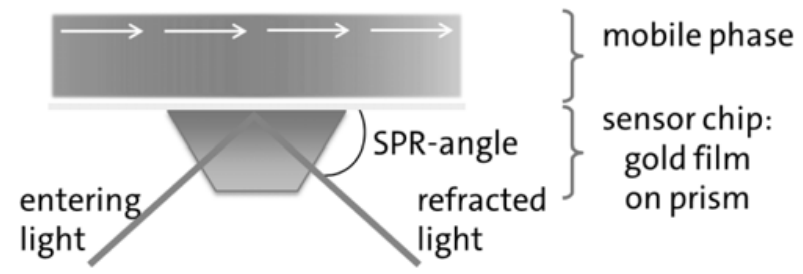

Figure 1. Assembly of SPR-sensor chip with the light path, gold film (between prism and mobile phase), mobile phase (on top with arrows), and prism (trapezoidal). The immobilized binding partner is located on the gold film in the mobile phase.

stabilized by ambient conditions like $\mathrm{pH}$, ionic strength or temperature [16]. Following the lock and key model, the aptamers can exhibit high affinity and specificity towards a wide range of molecules. An identification of potential candidates is conducted through a SELEX (systematic evolution of ligands by exponential enrichment), which must be performed one time. A broad variety of different procedures have been described in the literature since 1990, but the basic principles remain the same $[17,18]$. The starting point is always an aptamer library with a high number of nucleic acids which are composed of a randomized region flanked by constant primer regions. This aptamer pool and the target are incubated under the desired binding conditions so that a noncovalent bond between the potential binding partners can be established. Afterwards the non-binding respectively weak-binding aptamers are washed away. After this, the interaction between the promising candidates and the target is disrupted by harsher chemical conditions. After amplification, a new aptamer pool for the following round is generated. After 10-20 rounds, the diversity of aptamers is reduced to less aptamers and the final pool can be sequenced and the identified aptamers can be synthesized.

\section{Material and methods}

\subsection{Preparation of sensor chips}

The complete procedure was performed using a SPR4 (Sierra Sensors GmbH, Hamburg, Germany). The immobilization of proteins on the chip was possible due to a carboxyl-modification of the gold film. Covalent coupling was realized with terminal amino groups so that stable carboxamide bonds were established.

\subsubsection{Antibody-based biosensor}

After activation of the sensor chips by an injection of EDC/NHS (1-ethyl-3-(3-dimethylaminopropyl)carbodiimide/N-hydroxysuccinimide), the antibody (rabbit antilysozyme) was injected. Remaining binding sites on the gold film were blocked by an injection of ethanolamine over the complete surface. The chip can be stored in PBS and airtight sealed at $4{ }^{\circ} \mathrm{C}$ for at least 8 weeks.

\subsubsection{Aptamer-based biosensor}

For the studies with an aptamer-based sensor recently published aptamers with an affinity towards lysozyme have been used [18]. The SELEX was conducted using a white wine simulating buffer whose ion composition and $\mathrm{pH}$ are comparable to white wine.

After activation of the sensor chip as described above, streptavidin was injected. Since the interaction between streptavidin and biotin is one of the strongest and very specific, it is possible to immobilize $5^{\prime}$-biotinylated aptamers strictly orientated. For this purpose, the aptamers were 5'-modified using BEAMing (see below). The resulting biotinylated aptamers were injected directly into the SPR-system on one of the four spots on the chip. Remaining binding sites on the gold film were blocked by an injection of ethanolamine and biotin over the complete surface.

\subsection{Antibody-based biosensor measurement and data evaluation}

During the measurements PBS-T (PBS $+0.05 \%$ Tween ${ }^{\circledR} 20$ ) was used as running buffer. Every measurement cycle starts with the injection of analyte solution followed by a dissociation time in which unspecific bound molecules dissociate. After that, the sensor needs to be regenerated for next measurement which is completed by a few injections of an alkaline buffer with high ionic strength. Evaluation of the obtained sensogramms was performed using Analyzer R2-software (Sierra Sensors GmbH, Hamburg, Germany). Every signal was referenced against ethanolamine (blocking reagent). For all calculations the average of three data points after the end of the injection (during the dissociation time) was used (see Fig. 3). Only curves with a stable progression parallel to the $\mathrm{x}$-axis indicate a specific interaction between the binding partners. The calibrations curves were fitted with OriginPro 9.0G using four parameter regression.

\subsection{Aptamer-based biosensor measurement and data evaluation}

During the measurements white wine simulating buffer $(0.05 \%$ Tween $\mathrm{R} 20)$ was used as running buffer. Every measurement cycle starts with the injection of analyte solution followed by a dissociation time in which unspecific bound molecules dissociate. After that, the sensor needs to be regenerated for the next measurement which is completed by a short injection of an alkaline buffer with high ionic strength. The evaluation of the obtained sensogramms was performed using Analyzer R2software (Sierra Sensors GmbH, Hamburg, Germany).

\subsection{BEAMing}

The modification of aptamers can be performed by a PCR with modified primers. Since the PCR-products are double-stranded, a strand separation needs to be performed to isolate the single-stranded aptamers. Both requirements can be fulfilled by the use of BEAMing (Beads, Emulsion, Amplification, Magnetic), a combination of emulsion-PCR and solid phase-coupling according to Diehl and Hünniger $[18,19]$. Briefly, an according to the requirements modified Forward-Primer $\left(5^{\prime}\right.$-aminated or 5'-biotinylated), a Reverse-Primer coupled to magnetic beads and the other PCR components (polymerase, buffer, dNTPs, template and water) were mixed. After that, an emulsion was prepared by the addition of oil components so that various small reaction rooms with 
Table 1. Properties of the investigated white wines.

\begin{tabular}{|c|c|}
\hline alcohol content [vol.-\%] & grapes \\
\hline 0.0 & cuvee \\
\hline 9.5 & cuvee \\
\hline 11.5 & Müller-Thurgau \\
\hline
\end{tabular}

all necessary reaction components were created. After the amplification procedure, the double stranded PCRproducts were immobilized via the Reverse-Primer at the magnetic particles. By a heat denaturation and magnetic separation, an isolation of the then modified aptamers can be realized (see Hünniger [18] for detailed information).

\section{Results and discussion}

\subsection{Antibody-based biosensor}

The gold standard for the detection of allergens is currently the ELISA. Due to the high affinity and specificity of the antibodies this method combines a high sensitivity with a direct proof of the allergenic epitopes. Recent developments in legislation require a reduction or relinquishment of antibodies. A possibility to achieve this is the development of techniques which enable a multiple use of antibodies. The SPR-technology is a flowbased system in which one of the binding partners, in this case the antibody, is immobilized on a sensor chip. This chip is reusable multiple times so that the overall consumption of the antibody is significantly reduced. Since the quantification is performed by a change of the SPR-angle, it is not necessary to implement a secondary antibody [13].

In the presented work, a previously generated antibody against lysozyme was immobilized on the sensor chip surface. At first, lysozyme was diluted with PBS and injected into the system to check if the antibody is biologically active. The typical curve characteristics respectively elements of a SPR-measurement are clearly distinguishable (association and dissociation), so that dilution series of lysozyme were injected. In Fig. 2 the resulting sensogramms are displayed (unspecific bondings to the sensor chip surface were eliminated by referencing). A concentration dependency is clearly recognizable, so that multiple options for evaluation are available. For recording of SPR-curves like in Fig. 2, it is necessary that the running buffer is equivalent to the sample medium. Under these conditions both association and dissociation phase can be used for data evaluation.

Since the goal is the measurement of real samples, which possess different chemical properties, it is more advisable to use parts of the dissociation time for data evaluation. For this purpose, the mean of three data points in the middle of the dissociation can be used. A stable interaction between antibody and analyte is indicated by a dissociation curve which is parallel to the $\mathrm{x}$-axis.

After the binding of lysozyme in PBS by the immobilized antibody has been successfully completed, the next step is the examination of real samples. Therefore, commercially available wines have been spiked with decreasing amounts of lysozyme. As PBS was still used as running buffer and the used white wines differ substantially, not only but in particular, with regard to ion composition, $\mathrm{pH}$, polyphenol content and carbohydrates,

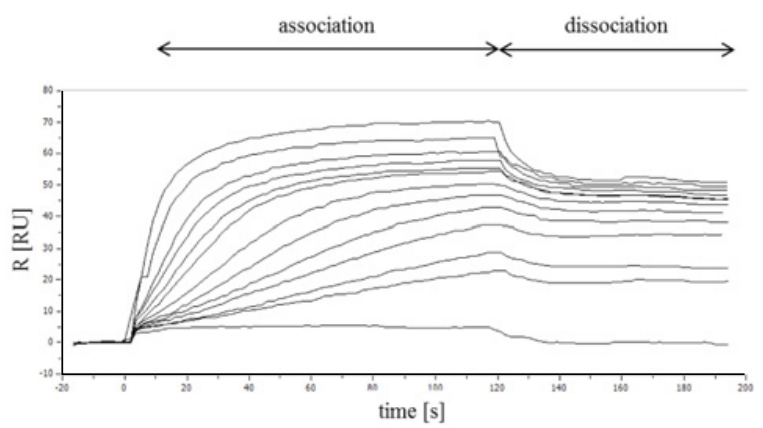

Figure 2. Antibody-based SPR-biosensor (immobilized lysozyme-antibody, running buffer PBS-T). Overlay of SPRsensogramms, every injection referenced to the blocked sensor chip surface. Dilution series $(0.013 \mathrm{ppm}, 0.020 \mathrm{ppm}, 0.025 \mathrm{ppm}$, $0.033 \mathrm{ppm}, 0.050 \mathrm{ppm}, 0.066 \mathrm{ppm}, 0.100 \mathrm{ppm}, 0.125 \mathrm{ppm}$, $0.160 \mathrm{ppm}, 0.200 \mathrm{ppm}, 0.330 \mathrm{ppm}, 0.500 \mathrm{ppm}$ ) of lysozyme in PBS. The characteristic elements of SPR-sensogramm are clearly visible: Association phase (0-125 s) and dissociation phase (125-185 s). Subsequently followed by the regeneration phase. $R[R U]=$ change of the SPR-angle.

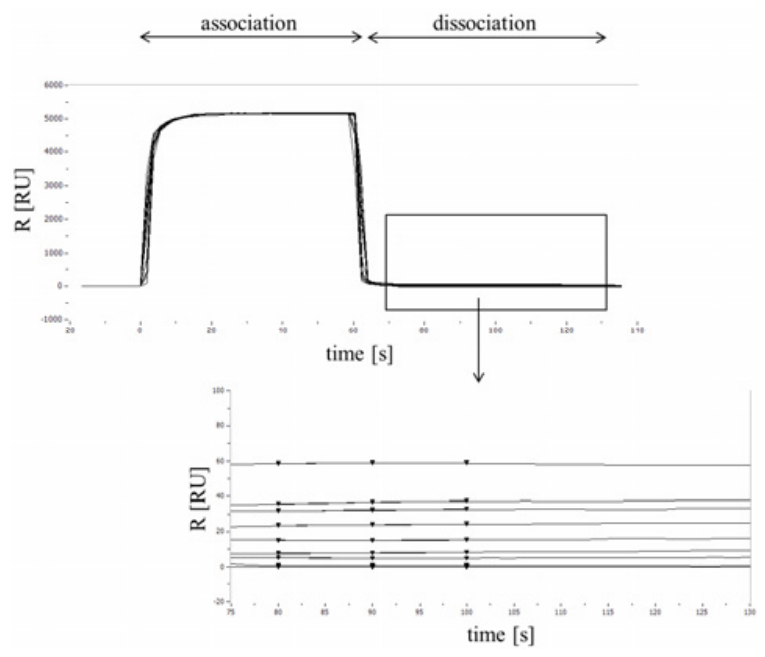

Figure 3. Visualization of real sample evaluation with antibodybased SPR-biosensor ( $25 \mu \mathrm{L}$ white wine (9.5 vol.-\%, cuvee), 60 s dissociation time, immobilized lysozyme-antibody, running buffer PBS-T). Samples spiked with lysozyme (0.05 ppm, 0.25 ppm, 0.5 ppm, 1 ppm, 2.5 ppm, 5 ppm, 10 ppm, 100 ppm) and referenced to blocked sensor chip surface. Top: SPRsensogramms of spiked wine samples. During the injection of samples (association) the typical curve progression is not observable. Bottom: Magnified dissociation time with three data points whose mean is used for the calculations. Concentration dependency is observable. $\mathrm{R}[\mathrm{RU}]=$ change of the SPR-angle.

the association phase does not result in an evaluable signal (Fig. 3, top). As described above, the mean of three data points during the dissociation time (Fig. 3, bottom) was used for all following calculations (referenced to blocked sensor chip surface).

The resulting data of three independent measurements were averaged and fitted using a four parameter logistic regression. This model is often used for calibration in immunoassays since it is both flexible and reliable [20]. To assess the quality of the method, the heteroskedasticity must be calculated for the whole curve progression 

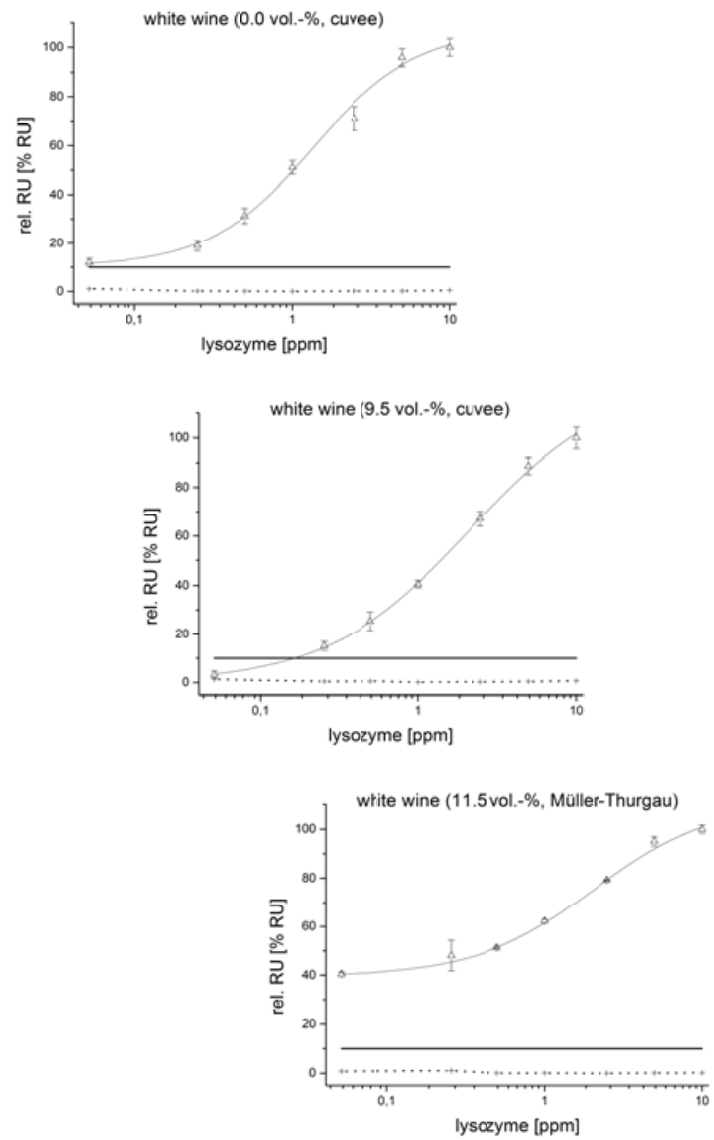

Figure 4. Matrix calibrations of three white wine samples spiked with lysozyme $(0.05 \mathrm{ppm}, 0.25 \mathrm{ppm}, 0.5 \mathrm{ppm}, 1 \mathrm{ppm}, 2.5 \mathrm{ppm}$, $5 \mathrm{ppm}, 10 \mathrm{ppm}$ ) and measured with antibody-based biosensor (immobilized lysozyme-antibody). For the evaluation was the mean of three data points during dissociation time used. Data $(n>3)$ were referenced to the blocked sensor chip surface and normalized to the highest concentration. Regression was performed using four parameter regression. Bold: maximum of $10 \%$ variance; dotted: variance over the whole curve progression, thin line: calculated regression. All data points with standard deviation.rel. RU [\% RU]= relative change of the SPR-angle, normalized to maximum signal.

(a value less than $10 \%$ was chosen as acceptable). Due to the sigmoidal curve progression, a calculation of the LOD and LOQ using multiples of the standard deviation is not advisable, so that the LOD and LOQ were estimated [10, 21]. For three different wines (see Table 1) it was possible to calculate calibrations curves in a range from $0.05 \mathrm{ppm}$ to $10 \mathrm{ppm}$, with a variance below $10 \%$ for the whole calibration range (see Fig. 4). This shows that the developed biosensor is capable to detect lysozyme in real samples even in amounts below the concentration of $0.25 \mathrm{ppm}$ which requires a labelling. A detection of even lower concentrations seems definitely achievable, since only $25 \mu \mathrm{L}$ sample were injected. With larger volumes results a longer injection time and therefore a longer time to build the antigen-antibody-bond.

The binding of other wine components than allergens should be avoided since this can lead to unnecessary labelling and therefore to limitations in the life of sensitive individuals. The developed biosensor was tested by various injections of different proteins (ovalbumin, caseins (alpha, beta, kappa), lactalbumines, ethanolamine).

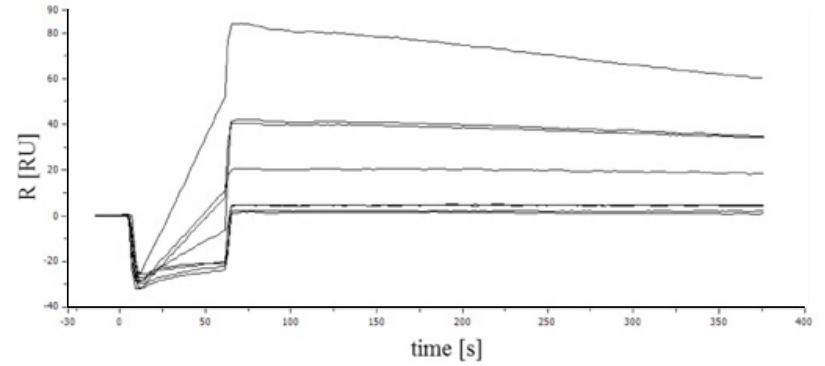

Figure 5. Aptamer-based SPR-biosensor (immobilized lysozyme-aptamer LysApt4 [18], running buffer white wine simulating buffer $+0.05 \%$ Tween-20) [18]. Overlay of SPR-sensogramms, dilution series of lysozyme (0.01 ppm, 0.03 ppm, 0.05 ppm, 0.08 ppm, 0.25 ppm, 0.5 ppm, 0.8 ppm, $1 \mathrm{ppm})$ in white wine simulating buffer.

After referencing to the blocked sensor chip, none of the other injections exhibited signal above zero. So, it can be concluded that the generated antibody-based biosensor is both sensitive and specific.

\subsection{Aptamer-based biosensor}

The next step to fulfill the requirements of the current legislation regarding the protection of animals for scientific purposes is the complete replacement of antibodies. Aptamers, single-stranded nucleic acids, could be an alternative to antibodies since the synthesis can be performed without the use of any animals or animalderived products. In the presented work, recently published aptamers with an affinity towards lysozyme have been used. The SELEX-procedure has been conducted in a buffer system which is in terms of ion composition and $\mathrm{pH}$-value comparable to white wine. As the secondary structure, and therefore also the affinity towards the target, is influenced by the surrounding medium, it is necessary to use the buffer also in the following investigations.

Starting point for the development of an aptamerbased biosensor was the immobilization of aptamers on the sensor chip surface. For this purpose, the commercially synthesized aptamers were modified by BEAMing to introduce a 5'-modification. The direct immobilization via an amine-modification was not successful since the aptamers do no longer show an affinity towards lysozyme. A more promising approach was the use of biotinylated aptamers and streptavidin so that the aptamers are more equally distributed over the surface. Furthermore, it seems reasonable to assume that no interactions between the aptamers can occur which would interrupt an aptamertarget interaction.

To test the developed aptamer-based biosensor, lysozyme in decreasing concentrations was injected into the SPR. Both as running buffer and for the dilution of the samples the white wine simulating buffer of the SELEX-procedure was used. The resulting sensogramms show that with higher concentrations an increase in the signal can be observed (Fig. 5). The analysis of real samples was not successful which is probably attributable to the dependence of the aptamer affinity on the buffer. Although the used buffer was designed to be in accordance with mean concentrations of ions in white wine, it is 
unlikely that the ion concentrations in the used wines comply exactly. So, it seems more constructive to select the aptamers in a buffer with high capacity, so that the influence of the individual sample can be reduced. In that approach, an ideal buffer composition for the stabilization of aptamers can be chosen since it is no longer necessary to align the conditions to white wine. Furthermore, it could be observed that the used aptamers do not show an absolute selectivity. The target, in this case lysozyme was preferably bound, but also other proteins of egg, e.g. ovalbumin, were retained by the immobilized aptamers. To increase the selectivity of the aptamers, it is necessary to improve the SELEXprocedure. Recent developments in this field of research indicate that a continuous monitoring of the SELEXprocess by techniques like next-generation sequencing is advisable [17]. Moreover, the use of bioinformatics to identify binding motives and the subsequent enhancement of affinity by the introduction of additional features (artificial nucleic bases or directed extension of the motives) are promising developments [22].

\section{Conclusion}

In the presented work, two approaches to minimize the use of antibodies were shown. The development of biosensors using the SPR-technology could be a possibility to fulfill the requirements of the EU directive 2010/63.

Especially the developed antibody-based biosensor could be an alternative, since it is reusable multiple times so that the overall consumption of antibodies can be reduced in comparison to the established ELISAtechnique. It is applicable in white wines and capable to detect lysozyme in concentrations which are even lower than demanded by the OIV $(0.25 \mathrm{ppm})$.

The aptamer-technology is a quite promising approach to replace antibodies completely, but more experiences are necessary to improve this technology. Overall, it should be taken into account that the detection of immunogenic characteristics is part where antibodies will keep an advantage.

An approach to overcome the limitations of both antibodies and aptamers could be the combination, e.g. in an ELISA-like format. If the allergen detection is performed by an antibody, it seems possible to develop an aptamer with an affinity towards the detection antibody.

\section{References}

[1] B. Y. Patel, G. W. Volcheck, Mayo Clin Proc. 90, 1411-1419 (2015)

[2] S. K. Sathe, C. Liu, V. D. Zaffran, Annu Rev Food Sci Technol. 7, 191-220 (2016)

[3] Regulation (EU) No 1169/2011 of the European Parliament and of the Council of 25 October 2011 on the provision of food information to consumers, amending Regulations (EC) No 1924/2006 and (EC) No 1925/2006 of the European Parliament and of the Council, and repealing Commission Directive 87/250/EEC, Council Directive
90/496/EEC, Commission Directive 1999/10/EC, Directive 2000/13/EC of the European Parliament and of the Council, Commission Directives 2002/67/EC and 2008/5/EC and Commission Regulation (EC) No $608 / 2004$

[4] Commission Implementing Regulation (EU) No $579 / 2012$ of 29 June 2012 amending Regulation (EC) No 607/2009 laying down certain detailed rules for the implementation of Council Regulation (EC) No 479/2008 as regards protected designations of origin and geographical indications, traditional terms, labelling and presentation of certain wine sector products

[5] OIV/OENO 427/2010 Criteria for the methods of quantification of potentially allergenic residues of fining agent proteins in wine

[6] Regulation (EU) No 1308/2013 of the European Parliament and of the Council of 17 December 2013 establishing a common organisation of the markets in agricultural products and repealing Council Regulations (EEC) No 922/72, (EEC) No 234/79, (EC) No 1037/2001 and (EC) No 1234/2007

[7] OIV-COMEX 502-2012 Revision of the limit of detection and limit of quantification related to potentially allergenic residues of fining agent proteins in wine

[8] J. Costa, P. Ansari, I. Mafra, M. B. P. P. Oliveira, S. Baumgartner, Anal Biochem Chem. 406, 2581-2590 (2014)

[9] R. M. Lequin, Clin Chem. 51, 2415-2418 (2005)

[10] M. Deckwart, C. Carstens, M. Webber-Witt, V. Schäfer, L. Eichhorn, S. Kang, M. Fischer, K. Brockow, M. Christmann, A. Paschke-Kratzin, J Agric Food Chem. 62, 6803-6812 (2014)

[11] R. Pilolli, A. Visconti, L. Monaci, Anal Biochem Chem. 407, 3787-3797 (2015)

[12] A. P. F. Turner, Biosens Bioelectron. 65, A1 (2015)

[13] R. Karlsson, J Mol Recogn. 17, 151-161 (2004)

[14] A. D. Ellington, J. W. Szostak, Nature. 346, 818-822 (1990)

[15] C. Tuerk, L. Gold, Science. 249, 505-510 (1990)

[16] M. McKeague, A. Giamberardino, M. C. DeRosa, Advances in aptamer-based biosensors for food safety (2011)

[17] T. Schütze, B. Wilhelm, N. Greiner, H. Braun, F. Peter, M. Mörl, V. A. Erdmann, H. Lehrach, Z. Konthur, M. Menger, P. F. Arndt, J. Glökler, PloS one. 6, e29604 (2011)

[18] T. Hünniger, H. Wessels, C. Fischer, A. PaschkeKratzin, M. Fischer, Anal Chem. 86, 10940-10947 (2014)

[19] F. Diehl, M. Li, Y. He, K. W. Kinzler, B. Vogelstein, Nat Methods. 3, 551-559 (2006)

[20] C. Carstens, M. Deckwart, M. Webber-Witt, V. Schäfer, L. Eichhorn, K. Brockow, M. Fischer, M. Christmann, A. Paschke-Kratzin, J Agric Food Chem. 62, 6247-6253 (2014)

[21] R. P. Ekins, Ligand Q. 4, (1981)

[22] P. Li, J. Sun, M. Su, X. Yang, X. Tang, Org Biomol Chem. 12, 2263-2272 (2014) 